\title{
Evaluación del Aporte de las Plantas Acuáticas Pistia stratiotes y Eichhornia crassipes en el Tratamiento de Aguas Residuales Municipales
}

\author{
Yoma I. Mendoza, Jhonny I.Pérez y Andres A. Galindo \\ Grupo de Investigación GISA, Facultad de Ingeniería, Universidad de La Guajira, Km 5 Vía a Maicao, \\ Riohacha-Colombia.E-mail: ymendoza@uniguajira.edu.co; jpemon@uniguajira.edu.co; \\ agalindo@uniguajira.edu.co
}

Recibido Jul. 17, 2017; Aceptado Oct. 5, 2017; Versión final Dic. 11, 2017, Publicado Abr. 2018

\begin{abstract}
Resumen
Se evaluó el aporte de las plantas acuáticas Pistia stratiotes y Eichhornia crassipes en el tratamiento del agua residual municipal (ARM) de la ciudad de Riohacha en Colombia. Se estableció un diseño experimental de un factor (plantas) y seis niveles que corresponden a los cinco tratamientos y un control. Se instalaron seis microcosmos con capacidad de $84,4 \mathrm{~L}$ que funcionaron a condiciones ambientales. De acuerdo a la prueba de Dunnet, no existe diferencia significativa entre los tratamientos y el control, por tanto no hay un aporte significativo de las plantas en la remoción de los contaminantes. Sin embargo, una pequeña diferencia en la remoción puede satisfacer las exigencias legales. Las máximas eficiencias de remoción se presentaron en los microcosmo con $100 \%$ y $50 \%$ cobertura de Pistia stratiotes, excepto $\mathrm{DBO}_{5}$ que se presentó en el sistemas con $50 \%$ de cada planta. Se alcanzaron remociones de: $78,5 \% \mathrm{NO}_{2}^{-}, 24,9 \%$ $\mathrm{NO}_{3}^{-}, 8,6 \% \mathrm{NH}_{4}^{+}, 51,6 \% \mathrm{PO}_{4}^{-3}, 79,1 \% \mathrm{DBO}_{5}, 76,2 \%$ DQO y $99,9 \%$ para $\mathrm{CT}$ y $\mathrm{CF}$.
\end{abstract}

\section{Evaluation of the Contribution of the Aquatic Plants Pistia stratiotes and Eichhornia crassipes in the Municipal Wastewater Treatment}

\begin{abstract}
The objective of this study was to evaluate the contribution of the aquatic plants Pistia stratiotes and Eichhornia crassipes in the treatment of municipal wastewater (ARM) in the city of Riohacha. An experimental design of a factor (plants) and six levels that correspond to the five treatments and a control were considered. Six microcosms with a capacity of $84.4 \mathrm{~L}$ that operated at environmental conditions were installed. According to the Dunnet test, there is no significant difference between treatments and control, so there is no significant contribution of plants in the removal of contaminants. However, a small difference in the removal can meet the legal requirements. The maximum removal efficiencies were presented in the microcosm with $100 \%$ and $50 \%$ coverage of Pistia stratiotes, except $\mathrm{BOD}_{5}$ that was presented in the systems with $50 \%$ of each plant. Removals of $78,5 \% \mathrm{NO}_{2}^{-}, 24,9 \% \mathrm{NO}_{3}^{-}, 8,66 \% \mathrm{NH}_{4}^{+}, 51,6 \% \mathrm{PO}_{4}^{-3}, 79,1 \%$ $\mathrm{DBO}_{5}, 76,2 \%$ DQO and $99,9 \%$ for CT and CF were achieved.
\end{abstract}

Keywords: phytoremediation; Pistia stratiotes; Eichhornia crassipes; pollutants; wastewater 


\section{INTRODUCCIÓN}

El agua residual está compuesta de una mezcla de materiales orgánicos e inorgánicos, suspendidos o disueltos, que provienen de una población después de haber sido modificadas por diversos usos en actividades domésticas, industriales y comunitarias (Diaz-Cuenca et al., 2012). El tratamiento de aguas residuales consiste en una serie de procesos que tienen como fin eliminar los contaminantes físicos, químicos y biológicos (González-Cabrera et al., 2014). Reducir la generación de aguas residuales y mejorar su tratamiento es una cuestión prioritaria a nivel mundial, ya que es importante disponer de agua de calidad y cantidad suficiente, lo que permitirá una mejora del ambiente, la salud y la calidad de vida (Romero et al., 2009). En América Latina y el Caribe solo el $20 \%$ de las aguas residuales municipales (ARM) son tratadas, en su mayoría con la tecnología de lodos activados o lagunas de estabilización (Hernández-Padilla et al., 2017). Sin embargo, se han realizado estudios que usan plantas acuáticas para el tratamiento de ARM, como una opción de bajos costos de operación y simplicidad en el manejo tecnológico, lo que se conoce como fitorremediación (Correa et al., 2015).

La fitorremediación es un tratamiento biológico que hace uso de las plantas y sus microorganismos asociados, se afirma que es un sistema eficaz, económico (en costos de operación y mantenimiento) y sostenible (Priyanka et al., 2017). La fitorremediación se puede lograr a través de diferentes procesos como la fitoextracción, rizofiltración, fitoestabilización, y fitotransformación/fitodegradación (Elias et al., 2014). El éxito de la fitorremediación depende principalmente de la actividad fotosintética y la tasa de crecimiento de las plantas (Vangronsveld et al., 2009). Hay una gran variedad de sistemas que usan plantas, algunas flotantes y otras con medios de soportes como los humedales. El uso de plantas acuáticas en el tratamiento de aguas residuales se ha convertido en un enfoque particular en los últimos años (Mumtaz et al., 2014; Vo et al., 2017). Las plantas acuáticas como Pistia stratiotes (lechuga de agua) y Eichhornia crassipes (jacinto de agua) han sido utilizadas para la eliminación de un amplio rango de contaminantes de las aguas residuales (Gupta et al., 2012; Rezania et al., 2014). La alta productividad de algunas plantas flotantes y el alto requerimiento nutricional de nitrógeno $(N)$ y fosforo $(P)$ hacen que estas especies resulten adecuadas para reducir los niveles de estos nutrientes de los efluentes (Sánchez, 2011).

Existe una gran variedad de plantas acuáticas usadas en los procesos de fitorremediación para remover contaminantes en ARM. Mudassar et al. (2014) ha evaluado la eficiencia de varias plantas (Pistia stratiotes, Eichhornia crassipess, Hydrocotyle umbellatta, Lemna minor, Tyhpa latifolia y Scirpus acutus) solas y combinadas. La Eichhornia crassipes y la Pistia stratiotes han sido ampliamente estudiadas por su potencial de fitorremediación para remover diversos contaminantes presentes en las aguas residuales como la demanda bilógica de oxígeno (DBO), demanda química de oxígeno (DQO), N y P (Akinbile y Yusoff, 2012; Sundaralingam y Gnanavelrajah, 2014). También se han usado la Eichhornia crassipess y Césped para remover diversos contaminantes presentes en ARM entre ellos los metales pesados (Rezania et al., 2015; Carreño y Granada, 2017). Al igual que se han usado Typha latifolia, Cyperus papyrus, Cyperus alternifolius y Pharagmites asutralis para remover patógenos como coliformes fecales (Kipasika et al., 2016).

La fitorremediación en humedales construidos usando macrófitos han logrado altas eficiencias en la reducción de la $\mathrm{DBO}$, nitrito $\left(\mathrm{NO}_{2}^{-}\right)$y fosfatos $\left(\mathrm{PO}_{4}^{-3}\right)$; estos sistemas son más eficaces en zonas tropicales donde la vegetación permanece todo el año (Fonkou et al., 2010). Sin embargo, a pesar de la importante función de las plantas acuáticas para remover los contaminantes de las ARM, se han encontrados casos donde las eficiencias son similares en sistemas sin y con plantas. Se citan algunos casos: en humedales construidos a escala piloto usando como medio de soporte la grava (Chan et al., 2008; Vera et al., 2010; Valipour et al., 2015), en humedales a escala de laboratorio (Yousefi y Mohseni-Bandpei, 2010; Aragón et al., 2015). De acuerdo a lo anterior, el objetivo de este trabajo es evaluar el real aporte de las plantas acuáticas Pistia stratiotes y Eichhornia crassipes, en la remoción de contaminantes de las ARM de la ciudad de Riohacha, departamento de La Guajira-Colombia. Para verificar el aporte de las plantas, se usó un diseño experimental de análisis de varianza de un factor o una vía (representada por las plantas) y 6 niveles que corresponden 5 tratamientos y un control.

\section{METODOLOGÍA}

A continuación se presentan los procedimientos metodológicos desarrollos en esta investigación, a saber:

Área de estudio; Diseño y esquema experimental, Agua residual municipal cruda; Aclimatación de las plantas y arranque del sistema; Procedimiento operacional; Muestreo y análisis en efluente y Análisis estadístico de los datos. 


\section{Área de estudio}

El estudio se realizó en la ciudad de Riohacha, zona costera ubicada al norte de Colombia, entre las coordenadas $11^{\circ} 33^{\prime} 15,65^{\prime \prime} \mathrm{N}$ y $72^{\circ} 55^{\prime} 26,09^{\prime \prime} \mathrm{W}$. En la ciudad prevalece un clima seco, con temperaturas que oscilan entre los $28^{\circ} \mathrm{C}$ a $45^{\circ} \mathrm{C}$, brillo solar de 6 a 7 horas de sol al día (radiación solar 5,5 a $6,0 \mathrm{Kwh} / \mathrm{m}^{2}$ ) y velocidades del viento de 5,7 a $11 \mathrm{~m} / \mathrm{s}$ (Rojano et al., 2016). Debido a las altas temperaturas y a la intensidad de los vientos la evaporación es muy alta, lo que produce la sensación térmica de intenso calor.

\section{Diseño y esquema experimental}

El sistema experimental estuvo integrado por seis unidades rectangulares de vidrio con dimensiones de 0,75 $\mathrm{m} \times 0,25 \mathrm{~m} \times 0,45 \mathrm{~m}$ (largo, ancho, alto) con un volumen total de 84,4 L y un volumen líquido de $40 \mathrm{~L}$, suficiente para generar una lámina de agua propicia para el desarrollo de las raíces y follaje de las plantas utilizadas. De acuerdo con lo indicado por Razania et al. (2016), la máximas eficiencias de remoción de los parámetros químicos, biológicos y físicos, se alcanzan dentro del rango de 13-17 días (el día 14 fue el óptimo), sin embargo para clima tropical un tiempo de retención de 7 días es suficiente para lograr un buen tratamiento y eliminación efectiva de los nutrientes (Olguín et al., 2017). Por lo anterior, el tiempo de retención evaluado en este estudio fue de 7 días, para mantener este tiempo constante fue necesario compensar diariamente con agua desionizada el volumen perdido por la transpiración de las plantas y la evaporación tal como lo hizo Priyanka et al. (2017). El sistema se instaló en el área posterior del laboratorio del Instituto de Estudios Ambientales y Aprovechamiento de Agua (INESAG) de la Universidad de La Guajira. El estudio se desarrolló a temperatura ambiente $\left(25\right.$ a $\left.38^{\circ} \mathrm{C}\right)$.

Se utilizaron dos especies de plantas acuáticas flotantes, Pistia stratiotes y Eichhornia crassipes. Estas plantas fueron seleccionadas por sus eficientes mecanismos para llevar a cabo los procesos de fitorremediación (Akinbile y Yusoff, 2012; Mumtaz et al., 2014). Se utilizó un diseño experimental de un factor (plantas) con 6 niveles que representan 5 tratamientos con coberturas de plantas y un control (sin plantas) así: (i) 50\% con Pistia stratiotes (M1); (ii) 50\% con Eichhornia crassipes (M2); (iii) 100\% combinando 50\% de cada planta (M3); (iv) 100\% con Pistia stratiotes (M4); (v) 100\% con Eichhornia crassipes (M5); y (vi) control, sin plantas (M6). Los sistemas con cobertura de $100 \%$ contenían 16 plantas de la misma especie y 8 plantas para los de $50 \%$.

\section{Agua residual municipal cruda}

El ARM afluente se recolectó en la estación de bombeo $N^{\circ} 2$ de la ciudad de Riohacha, la cual envía estos residuos al mar sin tratamiento previo. La recolección del ARM se realizaba semanalmente y se transportaban hasta el laboratorio del INESAG para su caracterización física, química y microbiológica, y alimentar los sistemas.

\section{Aclimatación de las plantas y arranque del sistema}

Las plantas utilizadas en este estudio fueron recolectadas en un jagüey (laguna construida) ubicado en el área urbana de la ciudad de Riohacha. Las raíces de las plantas se lavaron con agua de grifo para eliminar suciedad adherida. El periodo de arranque fue de 40 días. En los primeros 10 días las plantas fueron adaptas al sustrato usando recipientes plásticos de $30 \mathrm{~L}$. Durante este tiempo se varió cada 2 días la dilución del ARM al 20\%, 40\%,60\%, 80\% y 100\%. Este periodo fue fundamental para determinar el buen crecimiento de las plantas, seleccionando aquellas que tenían una altura de $25 \mathrm{~cm}$, medidas desde la raíz hasta la copa y un diámetro de $15 \mathrm{~cm}$ medido por la parte más ancha del follaje. Este tamaño garantizó una distribución apropiadas de las plantas en los microcosmos durante todo el periodo de estudio, especialmente aquellos que contenían 16 plantas. Las plantas seleccionadas fueron agregadas a los microcosmos de acuerdo al diseño experimental para su adaptación, desarrollo de las raíces y condiciones estables durante 30 días, tiempo en el cual se observó un buen desarrollo radicular (12 cm aprox.) y una remoción de DQO estable en $65 \%$.

\section{Procedimiento operacional}

Logrado el periodo de arranque, se inició la operación del sistema en los meses de mayo, junio y julio de 2015 durante 84 días. Las muestras fueron tomadas y analizadas cada 7 días, para un total de 12 muestras en cada microcosmo. Las unidades de tratamiento operaron a flujo semicontinuo, aplicándose tasa de renovación del efluente de $50 \%$ en cada TRH. Es decir, cada 7 días se extraían $20 \mathrm{~L}$ de efluente tratado y se reponían con ARM cruda; este mismo procedimiento fue aplicado en el estudio desarrollado por Mendoza et al. (2016). Las hojas secas eran retiradas y las plantas marchitas eran remplazadas, las raíces de las plantas jóvenes tienen mayor capacidad para absorber las impurezas y liberar oxígeno que las viejas (Tangahu et al., 2011). 


\section{Muestreo y análisis en efluente}

Los parámetros de control se determinaron diariamente in situ: temperatura, $\mathrm{pH}$ y oxígeno disuelto (OD), y en laboratorio se determinaron por triplicado ocho parámetros de funcionamiento: nitrito $\left(\mathrm{NO}_{2}^{-}\right)$, nitrato $\left(\mathrm{NO}_{3}^{-}\right)$, amonio $\left(\mathrm{NH}_{4}^{+}\right)$, fosfato $\left(\mathrm{PO}_{4}^{-3}\right), \mathrm{DBO}_{5}, \mathrm{DQO}$, y número más probable (NMP) de bacterias coliformes fecales (CT) y totales (CF). Los mismos parámetros fueron medidos en afluente y efluente. Para la recolección, preservación y análisis de las muestras se siguieron los procedimientos establecidos en el Método Estándar (APHA et al., 2012).

\section{Análisis estadístico de los datos}

El análisis se realizó usando el software estadístico IBM SPSS versión 21 (licencia Universidad de La Guajira). Para conocer la significancia de los resultados de cada parámetro operacional se realizó un análisis de varianza (ANOVA) de una vía con un nivel de significancia de $95 \%(p<0,05)$. Para determinar el aporte de las plantas se realizó una prueba a posteriori de Dunnet que compara los tratamientos y el control y se complementó con el test de Tukey para comparar las medias de todos los tratamientos. Las eficiencias de remoción fueron determinadas usando la ecuación 1 (Valipour et al., 2015).

$$
\text { Eficiencia de remoción }(\%)=\frac{\mathrm{Ca}-\mathrm{Ce}}{\mathrm{Ca}} * 100
$$

Donde: $\mathrm{Ca}=$ concentraciones en el afluente, $\mathrm{Ce}=$ concentraciones en el efluente.

\section{RESULTADOS Y DISCUSIÓN}

Los promedios de las características del ARM (Tabla 1) durante el período de estudio coincide con valores típicos de ARM de mediana concentración y con los valores del agua residual domestica utilizada en el estudio realizado por Valipour (2014). Esto es coherente dado que en la ciudad de Riohacha no existe presencia de industrias, por tantos sus residuos líquidos son domésticos, comerciales e institucionales. En temporadas de lluvias se pueden encontrar concentraciones de contaminantes menores debido a la mezcla de las aguas lluvias con las residuales. Las características encontradas en el ARM garantizaron el funcionamiento fisiológico de los microorganismos y plantas, además la concentración de los nutrientes permiten suplir un adecuado requerimiento nutricional de acuerdo al contenido de carbono, nitrógeno y fósforo. La relación $\mathrm{DBO}_{5} / \mathrm{DQO}$ en el afluente fue de 0,60; la cual es adecuada para el tratamiento biológico de las ARM (Hidalgo y Mejía, 2010).

\section{Niveles de remoción en los microcosmos}

En la Tabla 1 se pueden observar los resultados de los parámetros de control y operacionales obtenidos en los diferentes sistemas experimentales. Los valores medios se acompañan de la desviación estándar $(\overline{\mathrm{x}} \pm \sigma)$ en el afluente y efluente. La temperatura promedio es similar en todos los microcosmos variando con las condiciones climáticas, incluso horarios. El pH se mantuvo en el rango alcalino siendo menor en los microcosmos con plantas, lo que puede deberse al consumo de $\mathrm{CO}_{2}$ resultados de la fotosíntesis. Las bajas concentraciones de OD, se deben a la poca reaireación y a su contenido en el afluente. Un pH de 6-9 y temperatura de $15-38^{\circ} \mathrm{C}$ son favorable para el tratamiento de aguas residuales por plantas acuáticas (Mumtaz et al., 2014). Como es normal el oxígeno disuelto fue mayor en el efluente, el agua gana oxígeno a medida que se remueven los contaminantes debido a la aireación y el proceso de fotosíntesis. En los procesos aerobios, es primordial mantener una concentración adecuada de oxígeno disuelto, generalmente mayor de $1 \mathrm{mg} / \mathrm{L}$, valores inferiores afectan la eficiencia del tratamiento (Vymazal, 2011). El análisis estadístico de los resultados permitió establecer que no existe diferencia significativa $(p>0,05)$ entre los promedios de concentraciones evaluadas en el efluente de los diferentes microcosmos. De la misma manera, según la prueba de Dunnet no existe diferencia significativa entre los sistemas de tratamiento con plantas y el control (sin plantas). De acuerdo al test de Tukey no existe diferencia significativa entre ninguno de los microcosmos. Se puede decir entonces que las plantas no tienen un aporte estadísticamente significativo, sin embargo; en el cumplimiento de las exigencias ambientales una pequeña disminución en la concentración de un contaminante puede hacer que tal requerimiento se cumpla, especialmente en aquellos nutrientes difícil de remover como $\mathrm{NO}_{3}^{-}$y $\mathrm{PO}_{4}^{-3}$.

\section{Remoción de nutrientes: nitrógeno y fósforo}

Los microcosmos M1, M4 y M5 registraron valores promedio de $\mathrm{NO}_{2}^{-}$por debajo de $0,30 \mathrm{mg} / \mathrm{L}$ (Figura 1a) y porcentajes de remoción hasta $78,5 \%$, seguido del microcosmo M6 (control) donde se obtuvo un valor de 0,28 $\mathrm{mg} / \mathrm{L}$ y remociones de $55,7 \%$. En contraste con los microcosmos $\mathrm{M} 2$ y M3 que registraron concentraciones de $\mathrm{NO}_{2}^{-}$superiores a $0,30 \mathrm{mg} / \mathrm{L}$ y eficiencias inferiores al $50,0 \%$. Las concentraciones de $\mathrm{NO}_{3}^{-}$estuvieron por 
debajo de 1,4 mg/L (Figura 1b), mientras la eficiencia de remoción fue menor al 24,9\%, para todos los microcosmos siendo ligeramente mayor a las registradas en el control que fue de $11,5 \%$. $\mathrm{El} \mathrm{NH}_{4}^{+}$presentó concentraciones poco variables, en M1, M4, M5 y M6 se registraron valores cercanos a 0,6 mg/L y en M2 y $\mathrm{M} 3$, valores menores a $0,95 \mathrm{mg} / \mathrm{L}$ tal como se puede observar en la Figura 1c. En el microcosmos M2 y M3 no hubo remoción (-) de $\mathrm{NH}_{4}^{+}$, por el contrario se observó una acumulación, posiblemente porque las bacterias autótrofas no lo oxidan, generando eficiencias negativas. En general las eficiencias de remoción del $\mathrm{NH}_{4}^{+}$ fueron muy bajas (menores a 8,6\%). Como se observa en la Figura. 1a, 1b y $1 \mathrm{c}$ en algunos microcosmos existen concentraciones de $\mathrm{NO}_{2}^{-}, \mathrm{NO}_{3}^{-}$y $\mathrm{NH}_{4}^{+}$mayores o similares al afluente, lo que se explica por el ciclo del nitrógeno, el nitrógeno orgánico presente en ARM fresca se descompone en nitrógeno $\mathrm{NH}_{4}^{+}, \mathrm{NO}_{2}^{-}$y $\mathrm{NO}_{3}^{-}$, estos últimos aparecen y se incrementan con el paso de los días (Denk et al., 2017).

Tabla 1: Resumen estadístico de los datos en el afluentes y efluentes, y eficiencias de remoción en los tratamientos durante el periodo experimental $(\mathrm{n}=12$, media $\pm \sigma)$

\begin{tabular}{|c|c|c|c|c|c|c|}
\hline \multirow[b]{2}{*}{$\begin{array}{l}\text { Parámetros } \\
(\mathrm{mg} / \mathrm{L})\end{array}$} & \multicolumn{6}{|c|}{ Sistemas Experimentales } \\
\hline & $\begin{array}{c}\text { M1: } \\
\text { Microcosmos } \\
\text { con } 50 \% P . \\
\text { stratiotes }\end{array}$ & $\begin{array}{c}\text { M2: } \\
\text { Microcosmos } \\
\text { con } 50 \% E . \\
\text { crassipes }\end{array}$ & $\begin{array}{c}\text { M3: } \\
\text { Microcosmo100 } \\
\% \text { combinando } \\
50 \% \text { de cada } \\
\text { planta } \\
\end{array}$ & $\begin{array}{c}\text { M4: } \\
\text { Microcosmos } \\
\text { con } 100 \% P \\
\text { stratiotes } \\
\end{array}$ & $\begin{array}{c}\text { M5: } \\
\text { Microcosmos } \\
\text { con } 100 \% \text { E. } \\
\text { crassipes }\end{array}$ & $\begin{array}{c}\text { M6: } \\
\text { Microcosmos } \\
\text { de Control o } \\
\text { sin plantas }\end{array}$ \\
\hline $\begin{array}{c}\mathrm{pH} \\
\mathrm{A}(\overline{\mathrm{x}} \pm \sigma) \\
\mathrm{E}(\overline{\mathrm{x}} \pm \sigma) \\
\end{array}$ & $\begin{array}{l}7,60 \pm 0 \\
7,87 \pm 0\end{array}$ & $\begin{array}{l}7,60 \pm 0,16 \\
7,92 \pm 0,28 \\
\end{array}$ & $\begin{array}{l}7,60 \pm 0,16 \\
7,60 \pm 0,13 \\
\end{array}$ & $\begin{array}{l}7,60 \pm 0,16 \\
7,66 \pm 0,24\end{array}$ & $\begin{array}{l}7,60 \pm 0,16 \\
7,76 \pm 0,24\end{array}$ & $\begin{array}{l}7,60 \pm \\
8,29 \pm\end{array}$ \\
\hline $\begin{array}{c}T \\
A(\bar{x} \pm \sigma) \\
E(\bar{x} \pm \sigma)\end{array}$ & $\begin{array}{l}95 \\
28\end{array}$ & $\begin{array}{l}26,7 \pm 2,95 \\
26,4 \pm 2,44\end{array}$ & $\begin{array}{l}26,7 \pm 2,95 \\
26,5 \pm 2,09\end{array}$ & $\begin{array}{l}26,7 \pm \\
26,4 \pm\end{array}$ & $\begin{array}{l}26,7 \pm 2,95 \\
26,5 \pm 2,65\end{array}$ & $\begin{array}{l}26,7 \pm \\
26,3 \pm\end{array}$ \\
\hline $\begin{array}{r}\text { OD } \\
A(\bar{x} \pm \sigma) \\
E(\bar{x} \pm \sigma)\end{array}$ & $1,19 \pm$ & $1,19 \pm 0,79$ & $\begin{array}{l}1,19 \pm 0,79 \\
1,90 \pm 1,27\end{array}$ & $\begin{array}{l}1,19 \pm \\
1,79 \pm\end{array}$ & $\begin{array}{l}1,19 \pm 0,79 \\
2,43 \pm 1,77\end{array}$ & $\begin{array}{l}1,19 \pm \\
2,76 \pm\end{array}$ \\
\hline $\begin{array}{l}\quad \mathrm{NO}_{2}^{-} \\
\mathrm{A}(\overline{\mathrm{x}} \pm \sigma) \\
\mathrm{E}(\overline{\mathrm{x}} \pm \sigma) \\
\% \text { Remoción }\end{array}$ & $\begin{array}{c}0,633 \pm 1,490 \\
0,173 \pm 0,198 \\
72,6\end{array}$ & $\begin{array}{c}0,633 \pm 1,490 \\
0,413 \pm 0,383 \\
34,7 \\
\end{array}$ & $\begin{array}{c}0,633 \pm 1,490 \\
0,326 \pm 0,378 \\
48,4 \\
\end{array}$ & $\begin{array}{c}0,633 \pm 1,490 \\
0,136 \pm 0,274 \\
78,5\end{array}$ & $\begin{array}{c}0,633 \pm 1,490 \\
0,233 \pm 0,281 \\
63,1 \\
\end{array}$ & $\begin{array}{c}0,633 \pm 1,490 \\
0,28 \pm 0,652 \\
55,7 \\
\end{array}$ \\
\hline $\begin{array}{l}\quad \mathrm{NO}_{3}^{-} \\
\mathrm{A}(\overline{\mathrm{x}} \pm \sigma) \\
\mathrm{E}(\overline{\mathrm{x}} \pm \sigma) \\
\% \text { Remoción } \\
\end{array}$ & $\begin{array}{c}1,554 \pm 1,694 \\
1,166 \pm 0,784 \\
24,9 \\
\end{array}$ & $\begin{array}{c}1,554 \pm 1,694 \\
1,234 \pm 0,778 \\
20,5 \\
\end{array}$ & $\begin{array}{c}1,554 \pm 1,694 \\
1,231 \pm 0,800 \\
20,7 \\
\end{array}$ & $\begin{array}{c}1,554 \pm 1,694 \\
1,242 \pm 0,877 \\
20,0 \\
\end{array}$ & $\begin{array}{c}1,554 \pm 1,694 \\
1,226 \pm 0,815 \\
21,1 \\
\end{array}$ & $\begin{array}{c}1,554 \pm 1,694 \\
1,376 \pm 0,849 \\
11,5 \\
\end{array}$ \\
\hline $\begin{array}{l}\quad \mathrm{NH}_{4}^{+} \\
\mathrm{A}(\overline{\mathrm{x}} \pm \sigma) \\
\mathrm{E}(\overline{\mathrm{x}} \pm \sigma) \\
\% \text { Remoción }\end{array}$ & $\begin{array}{c}0,658 \pm 1,353 \\
0,601 \pm 1,176 \\
8,6 \\
\end{array}$ & $\begin{array}{l}0,658 \pm \\
0,711 \pm\end{array}$ & $\begin{array}{l}0,658 \pm 1,353 \\
0,932 \pm 1,652\end{array}$ & $\begin{array}{c}0,658 \pm 1,353 \\
0,637 \pm 1,185 \\
3,1 \\
\end{array}$ & $\begin{array}{c}0,658 \pm 1,353 \\
0,612 \pm 0,159 \\
6,9 \\
\end{array}$ & $\begin{array}{c}0,658 \pm 1,353 \\
0,641 \pm 1,663 \\
2,5 \\
\end{array}$ \\
\hline $\begin{array}{l}\quad \mathrm{PO}_{4}^{-3} \\
\mathrm{~A}(\overline{\mathrm{x}} \pm \sigma) \\
\mathrm{E}(\overline{\mathrm{x}} \pm \sigma) \\
\% \text { Remoción } \\
\end{array}$ & $\begin{array}{r}2,975 \pm \\
1,438 \pm \\
51 \\
\end{array}$ & $\begin{array}{r}2,975 \pm \\
1,714 \pm \\
42 \\
\end{array}$ & $\begin{array}{c}2,975 \pm 2,457 \\
1,881 \pm 1,820 \\
36,7 \\
\end{array}$ & $\begin{array}{r}2,975 \\
1,574 \\
47 \\
\end{array}$ & $\begin{array}{c}2,975 \pm 2,457 \\
1,653 \pm 2,010 \\
44,4 \\
\end{array}$ & $\begin{array}{c}2,975 \pm 2,457 \\
2,142 \pm 1,550 \\
28,0 \\
\end{array}$ \\
\hline $\begin{array}{l}\mathrm{DBO}_{5} \\
\mathrm{~A}(\overline{\mathrm{x}} \pm \sigma) \\
\mathrm{E}(\overline{\mathrm{x}} \pm \sigma) \\
\% \text { Remoción }\end{array}$ & $\begin{array}{c}206 \pm 87 \\
53 \pm 18 \\
74,0 \\
\end{array}$ & $\begin{array}{c}206 \pm 87 \\
59 \pm 22 \\
71,2 \\
\end{array}$ & $\begin{array}{c}206 \pm 87 \\
43 \pm 28 \\
79,1 \\
\end{array}$ & $\begin{array}{c}206 \pm 87 \\
51 \pm 32 \\
75,3 \\
\end{array}$ & $\begin{array}{c}206 \pm 87 \\
44 \pm 29 \\
78,5 \\
\end{array}$ & $\begin{array}{c}206 \pm 87 \\
68 \pm 27 \\
66,9 \\
\end{array}$ \\
\hline $\begin{array}{c}\text { DQO } \\
A(\bar{x} \pm \sigma) \\
E(\bar{x} \pm \sigma) \\
\% \text { Remoción } \\
\end{array}$ & $\begin{array}{c}355 \pm 115 \\
87 \pm 41 \\
75,4 \\
\end{array}$ & $\begin{array}{c}355 \pm 115 \\
93 \pm 62 \\
73,8 \\
\end{array}$ & $\begin{array}{c}355 \pm 115 \\
127 \pm 76 \\
64,2 \\
\end{array}$ & $\begin{array}{c}355 \pm 115 \\
84 \pm 39 \\
76,2 \\
\end{array}$ & $\begin{array}{c}355 \pm 115 \\
89 \pm 40 \\
74,8 \\
\end{array}$ & $\begin{array}{c}355 \pm 115 \\
123 \pm 41 \\
65,2 \\
\end{array}$ \\
\hline $\begin{array}{r}C F \\
A(\bar{x} \pm \sigma)\end{array}$ & $53^{*} 10^{7} \pm 20^{*} 10^{7}$ & $53^{*} 10^{7} \pm 20^{*} 10$ & $53^{*} 10^{7} \pm 20^{*} 10^{7}$ & $53^{*} 10^{7} \pm 20^{*} 10^{7}$ & $53^{\star} 10^{7} \pm 20^{*} 10$ & $53^{*} 10^{7} \pm 20^{*} 10^{7}$ \\
\hline$E(\bar{x} \pm \sigma)$ & $84^{*} 10^{4} \pm 26^{*} 10^{5}$ & $49^{*} 10^{4} \pm 97^{*} 10$ & $70^{*} 10^{4} \pm 11 * 10^{5}$ & $31 * 10^{4} \pm 67 * 10^{4}$ & $70^{*} \frac{1}{5}+11^{*} 10$ & $59^{*} 10^{4} \pm 10^{*} 10^{5}$ \\
\hline$\%$ Remocic & 99,8 & 99,9 & 99,8 & 99,9 & 99,8 & 99,8 \\
\hline $\begin{array}{r}C T \\
A(\bar{x} \pm \sigma)\end{array}$ & $24^{*} 10^{7} \pm 62^{*} 10^{6}$ & $24^{*} \underset{6}{10^{7} \pm 62^{*} 10}$ & $24^{\star} 10^{7} \pm 62^{*} 10^{6}$ & $24^{*} 10^{7} \pm 62^{*} 10^{6}$ & $24^{*} 10^{7} \pm 62^{*} 10$ & $24^{*} 10^{7} \pm 62^{*} 10^{6}$ \\
\hline$\sigma)$ & $37^{\star} 10^{3} \pm 69^{\star} 10^{3}$ & $10^{*} 10^{4} \pm 11^{*} 10$ & $23^{*} 10^{4} \pm 51^{*} 10^{4}$ & $18^{*} 10^{3} \pm 11^{*} 10^{3}$ & $11^{*} 10^{4} \pm 14^{*} 10$ & $22^{*} 10^{4} \pm 38^{*} 10^{4}$ \\
\hline \% Remoci & 99,9 & 99,9 & 99,9 & 99,9 & 99,9 & 99,9 \\
\hline
\end{tabular}


Niveth et al. (2016) a escala laboratorio usando Pistia stratiotes lograron eficiencias de 60,3\% y 6,6\% para $\mathrm{NO}_{3}^{-}$, $63,2 \%$ y $10,4 \%$ para el $\mathrm{NH}_{4}^{+}$, en los sistemas con plantas y control respectivamente, determinando que existe diferencia significativa en las unidades con plantas y el control $(p<0.05)$. De manera semejante a escala piloto usando macrófita Typha dominguensis alimentado con el efluente de un sistema de lagunas de estabilización que trata ARM se alcanzaron eficiencia de 45,0 y $55,0 \%$ para $\mathrm{NH}_{4}^{+}$y 14,0 y $15,0 \%$ para $\mathrm{PO}_{4}^{-3}$ en el sistema con plantas y control, respectivamente. No se observó diferencia significativa $(p>0,05)$ en el sistema con planta y el control. Para el caso del $\mathrm{NH}_{4}^{+}$los justificaron indicando que la remoción puede estar relacionada más con el proceso de nitrificación y desnitrificación que con la presencia de las plantas (Vera et al., 2010).

Las concentraciones medias del $\mathrm{PO}_{4}^{-3}$ en el efluente fueron similares en los sistemas con plantas, encontrando valores por debajo de $2 \mathrm{mg} / \mathrm{L}$ (Figura 1d), el mejor comportamiento lo presentó M1 con porcentaje de remoción de $51,6 \%$. Los valores medios del control fueron ligeramente mayores $(2,5 \mathrm{mg} / \mathrm{L})$ y la eficiencia solo alcanzó a 28,0\%. De igual forma Yousefi y Mohseni-Bandpei (2010), realizaron un estudio a escala de laboratorio con humedales construidos de flujo horizontal subsuperficial empleando diferentes densidades de plantas (Iris pseudacorus), y un control tratando ARM. Las eficiencias de remoción para el $\mathrm{PO}_{4}^{-3}$ alcanzaron $74,9 \%$ para los sistemas con plantas, y $63,1 \%$, en el control, determinando que no hay una diferencia significativa en los sistemas con plantas y sin plantas $(p>0.05)$.
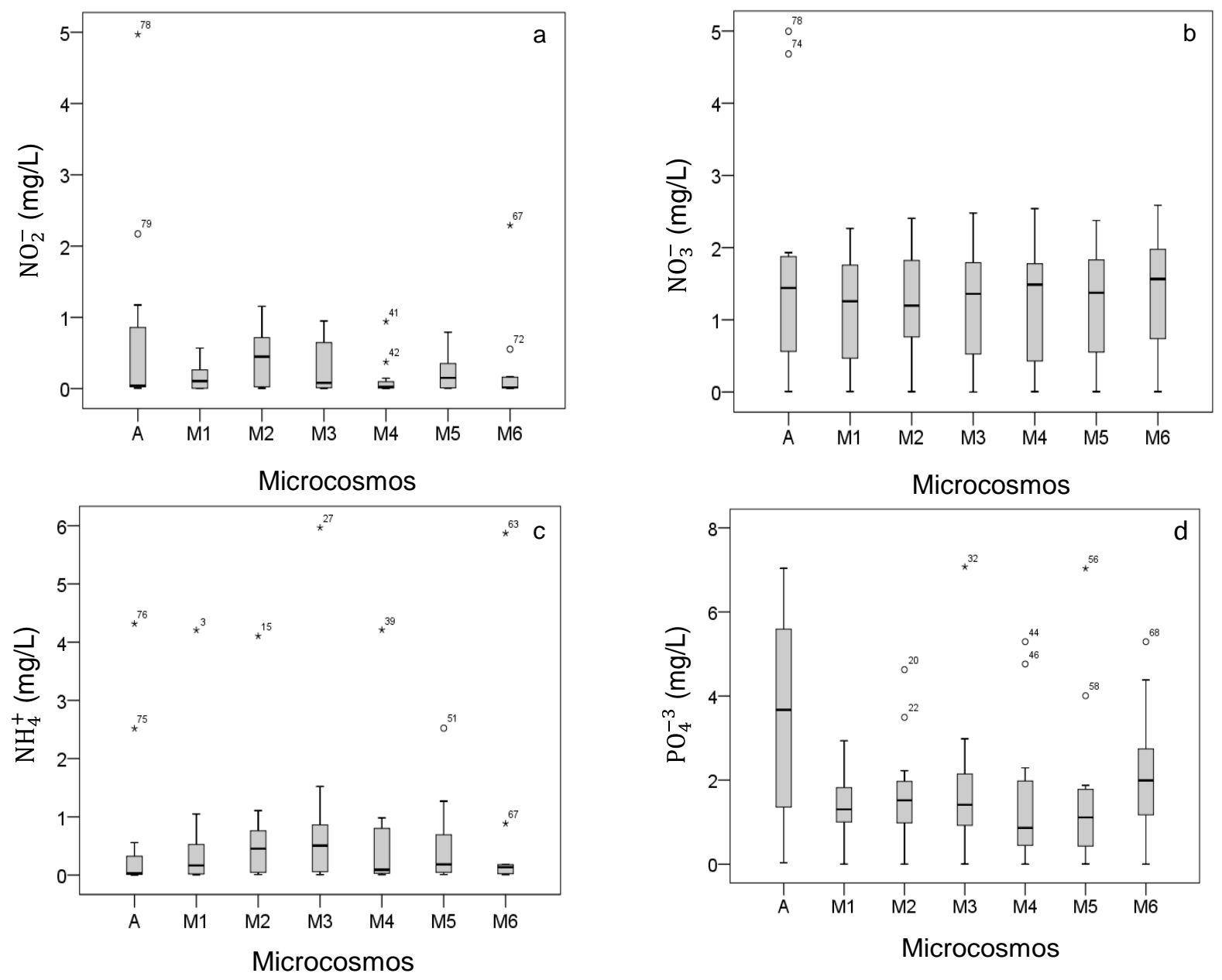

Fig. 1: Concentración final de Nitrito (a), Nitrato (b), Amonio (c) y Fosfato (d) en los diferentes microcosmos y el afluente.

Remoción de materia orgánica: $D B O_{5}$, y $D Q O$

En todos los sistemas de tratamiento (con y sin planta) se reflejó una marcada disminución de la $\mathrm{DBO}_{5}$ y DQO. Los microcosmos con plantas registraron eficiencias de remoción de 71,2 a $79,1 \%$, y 64,2 a $76,2 \%$ para la $\mathrm{DBO}_{5}$ y DQO respectivamente; valores cercanos fueron obtenidos en el control, con eficiencias de $66,9 \%$ para $\mathrm{DBO}_{5}$ y $65,2 \%$ para la DQO; lo que ratifica la no existencia de la diferencia significativa en los diferentes tratamientos. En la Figura 2 se puede ver el comportamiento en la remoción de la $\mathrm{DBO}_{5}$ (Figura 2a) y DQO (Figura 2b). Estudio realizado en humedales a escala de laboratorio Valipour et al. (2015) usando Eichhornia crassipes y Bio-Hedge reportaron una remoción del 79,0\% y $84,0 \%$ para DQO y DBO5 respectivamente. Contrario a los resultados del control donde se alcanzaron eficiencia del 25,0\% y 33,0\% para la DQO y DBO5 respectivamente. Esta diferencia puede deberse a un medio de soporte para fijar el sistema radicular de las 
plantas. Lekeufack et al. (2012) usaron Echinochloa pyramidalis a escala piloto y encontró una remoción del

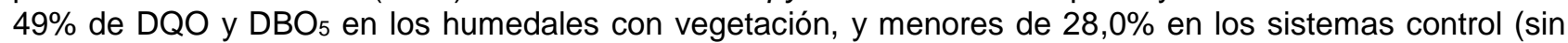
vegetación). El humedal con vegetación fue significativamente más eficiente $(p<0.05)$ que el control en la reducción de $\mathrm{DBO}_{5}$ y DQO; explicando que estas diferencias se deben al papel que juegan las raíces de las plantas en la matriz sustrato-raíz, especialmente cuando se adhieren a un medio de soporte.

Si bien no se encontraron diferencias significativas, algunos microcosmos presentan mayor remoción de la materia orgánica $\left(\mathrm{DBO}_{5}\right.$ y $\left.\mathrm{DQO}\right)$ que el control, se puede considerar que se debe a la eliminación por los microorganismos que viven adheridos al sistema radicular de las plantas (Sánchez, 2011). Por otro lado, la materia orgánica sedimentables es rápidamente eliminada en los sistemas por condiciones de reposo, mientras que la eliminación de la DBO soluble puede ser eliminada por microorganismos presente en el ARM (Mumtaz et al, 2014). Esto pudo ocurrir en el control de este estudio, debido a que la falta de plantas da lugar a la aireación por parte de la atmósfera, lo que conduce a la oxidación de la carga orgánica por organismos presentes (Vymazal y Kröpfelová, 2009) especialmente en condiciones de altas temperaturas que favorecen la reacción como es el caso de la ciudad de Riohacha.
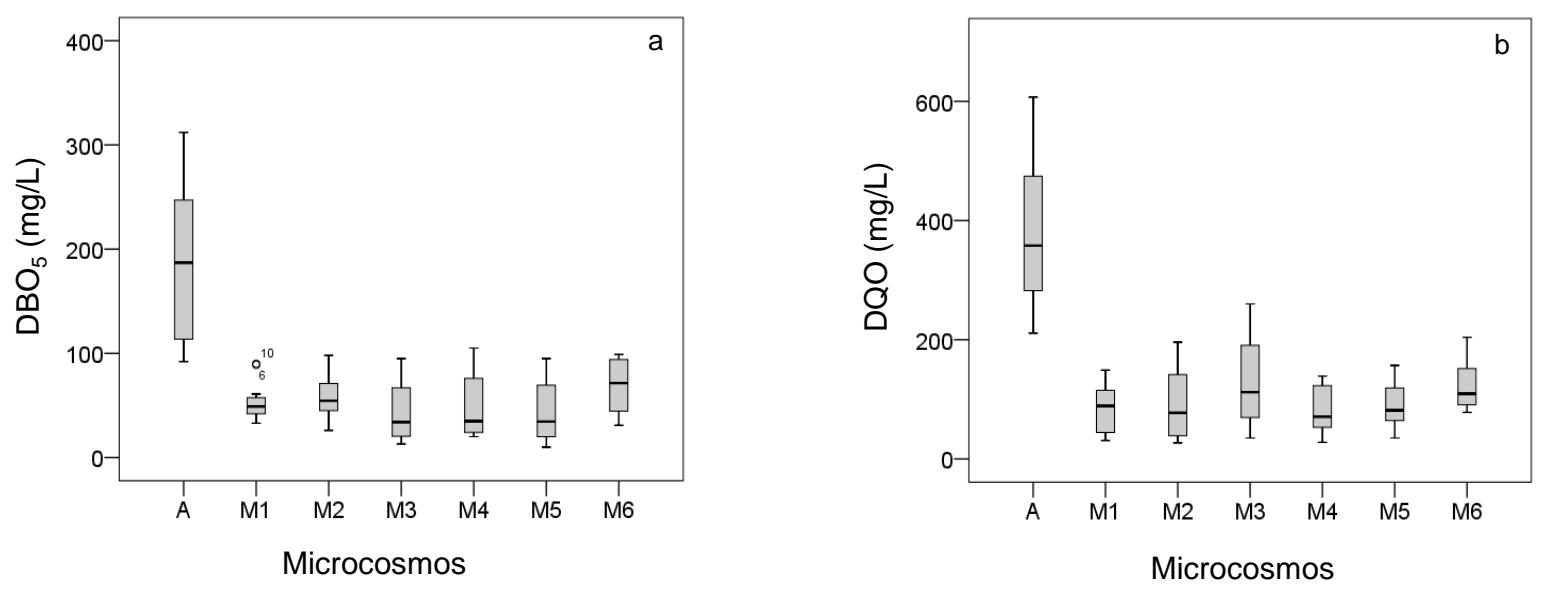

Fig. 2: Concentración de $\mathrm{DBO}_{5}(\mathrm{a})$ y $\mathrm{DQO}$ (b) en los diferentes microcosmos y el afluente

\section{Remoción de microorganismos: Coliformes Fecales y Coliformes Totales}

La concentración de coliformes en los efluentes de los diferentes sistemas de tratamientos fueron muy variados, las concentraciones promedio de CF variaron de $3,1^{*} 10^{5}$ a $8,4^{*} 10^{5}$ (Figura $3 a$ ) y las de CT de $1,8^{*} 10^{4}$ a $2,310^{5}$. (Figura 3b). Todos los sistemas (con y sin plantas) alcanzaron eficiencias superiores al 99,8\%. Lekeufack et al., (2012), realizó un estudio para evaluar la eliminación de microrganismos del ARM en humedales a escala piloto. Afirman no encontrar diferencias significativas en los CF y estreptococos; esta diferencias solo ocurre en los CT en época seca. Aragón et al. (2015) a escala piloto encontraron remoción de CT que alcanzaron 78,9\% en el control y $77,3 \%$ en los sistemas con plantas. A pesar de tener mejores resultados en el control no se existe diferencia significancia entre los resultados de las dos unidades. La eliminación de coliformes en el control se puede atribuir a la inactivación de los patógenos a temperatura superiores a $7,7^{\circ} \mathrm{C}$; los rayos ultravioletas hacen que los microorganismos en el agua presenten un cambio genético (ADN), haciendo imposible la generación de proteína que lo mantiene vivo y que permite su reproducción (Boutilier et al., 2009).
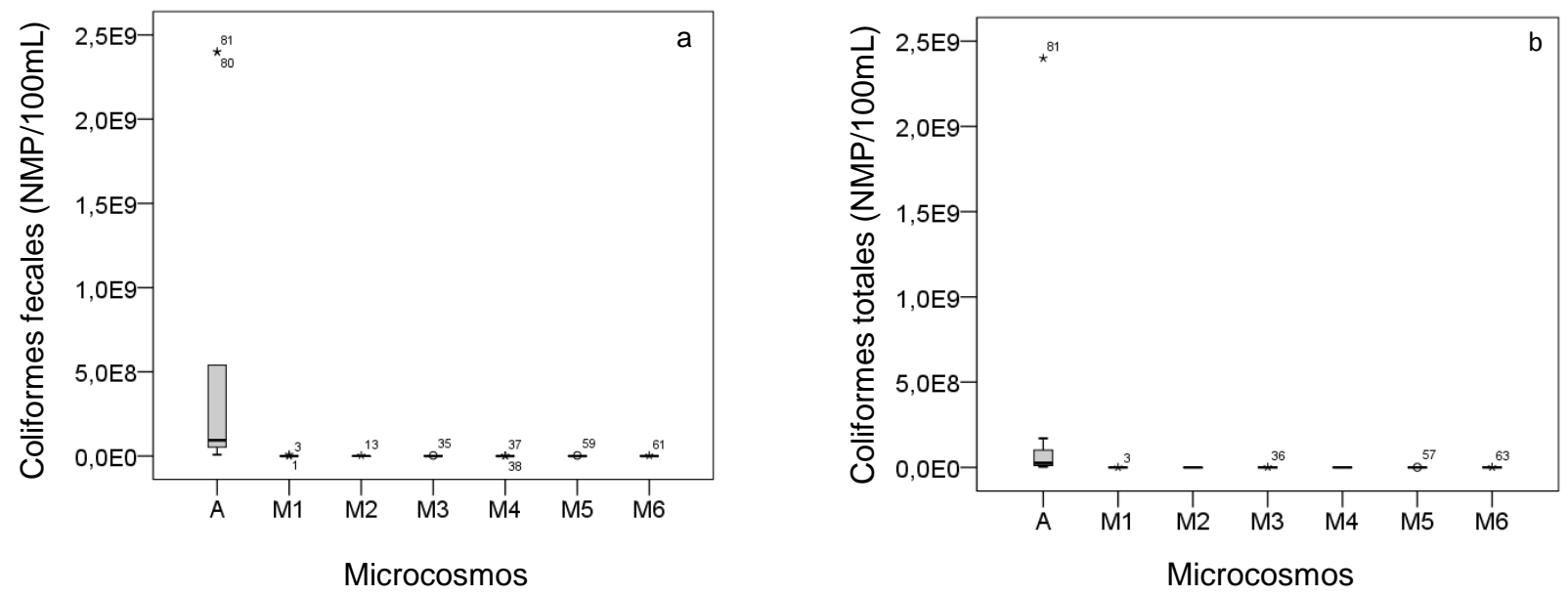

Fig. 3: Concentración de CF (a) y CT (b) en los diferentes microcosmos y el afluente 
Como se ha mencionado anteriormente no se encontraron diferencias significativas entre los tratamientos y el control. Sin embargo, analizando los resultados se pudo evidenciar que las mayores eficiencias de remoción se presentaron en los microcosmo con 100 y $50 \%$ cobertura de Pistia stratiotes, excepto $\mathrm{DBO}_{5}$ que se presentó en el sistemas con $50 \%$ de cada planta, donde se refleja la contribución de estas plantas reportadas en la literaturas como eficiente en la degradación de los contaminantes presentes en ARM (Mudassar et al., 2014; Akinbile y Yusoff, 2012). En este caso la no significancia estadística no resta la importancia de las plantas en el proceso de fitorremediación; las normas ambientales son cada vez más exigentes, por tanto una pequeña disminución en la concentración de los contaminantes, puede permitir el cumplimiento de estas exigencias legales.

\section{CONCLUSIONES}

1. De acuerdo a la prueba de Tukey no se registró diferencia significativa en ninguno de los microcosmos y de acuerdo a Dunnet tal diferencia no fue observada entre los sistemas de tratamiento y el control, lo que indica que para el tipo de sustrato y condiciones de temperatura no hubo aporte significativo de las plantas acuáticas utilizadas.

2. A pesar de no existir aporte significativa de las plantas, se encontró que las mejores eficiencias se lograron en el microcosmo que tenía el $100 \%$ de cobertura con Pistia stratiotes (M4), seguido del microcosmo que tenía el 50\% de cobertura con la misma especie (M1), y en un tercer lugar el microcosmo M5 (100\% Eichhornia crassipes). Sin embargo, en muchos casos un ligero aumento en la remoción de un contaminante puede ser muy importante para cumplir con los estándares ambientales en cualquier país.

3. Las eficiencias encontradas en los microcosmos especialmente aquellos con cobertura de Pistia stratiotes y Eichhornia crassipes alcanzaron remoción de $78,5 \% ; 79,1 \% ; 76,2 \%$ y $99,9 \%$ para $\mathrm{NO}_{2}^{-}$, $\mathrm{DBO}_{5}$, DQO y CT respectivamente; no siendo así para el $\mathrm{NH}_{4}^{+}, \mathrm{PO}_{4}^{-3}$ y NO $24,9 \%$, respectivamente. Para la remoción de CF y CT las eficiencias de remoción fueron superiores al $99 \%$ incluso en el control.

4. Independientemente del gran aporte de las plantas, en los humedales construidos se generan procesos remediadores; su facilidad de operación y bajo costo, proporcionan una alternativa viable de tratamiento de aguas residuales, al disminuir la carga orgánica contaminante en cantidades importantes

\section{AGRADECIMIENTOS}

Se agradece a la Universidad de La Guajira por la financiación y espacio físico para la instalación proyecto. A Margedis Oñate y Laura López por el trabajo de campo, laboratorio y organización de los datos que soporta esta publicación, y a los colaboradores del Instituto de Estudios Ambientales y Aprovechamiento de Agua (INESAG) de la Universidad de La Guajira. Agradecimientos también al profesor José Alberto Salgado Chavez de la Universidad de La Guajira por la revisión final del documento.

\section{REFERENCIAS}

Akinbile, C. y Yusoff, M., Assessingwater hyacinth (Eichhornia crassipes) and lettuce (Pistia stratiotes) effectiveness in aquaculture waste water treatment, doi: http://dx.doi.org/10.1080/15226514.2011.587482, Int. J. Phytoremediation, 14(3), 201-211 (2012)

Aragón R. Parra A. y Peña M., Evaluación Preliminar del Funcionamiento de Un Sistema Prototipo De Humedales Artificiales Empleando Heliconia psittacorum Y Cyperus papyrus Para El Tratamiento De Aguas Residuales, https://goo.gl/gWBA96, ISSN: 2322-9071, Agroecología Ciencia y Tecnología, 3(1), 7-12 (2015)

APHA, AWWA, WEF. Standard Methods for examination of water and wastewater. XXII Ed., 1360 p. American Public Health Association, Washington, USA (2012)

Boutilier, L., Jamieson, R., Gordon, R., Lake, C. y Hart, W., Adsorption, sedimentation, and inactivation of E. coli within wastewater treatment wetlands, doi: https://doi.org/10.1016/j.watres.2009.06.039, Water Research, 43, 4370-4380 (2009)

Carreño, U.F. y Granada, C.A., Design, development, and evaluation of a laboratory-scale phytoremediation system using Eichhornia crassipes for the treatment of chromiumcontaminated water, doi: http://dx.doi.org/10.18180/tecciencia.2017.22.2, TECCIENCIA, 12(22), 7-14 (2017)

Chan, SY., Tsang, YF., Cui, L.H. y Chua, H., Domestic wastewater treatment using batch-fed constructed wetland and predictive model development for $\mathrm{NH}_{3}-\mathrm{N}$ removal, doi: https://doi.org/10.1016/j.procbio.2007.12.009, Process Biochemistry, 43, 297-305 (2008)

Correa, S., Gamarra, Y., Salazar, A. y Pitta, N., Evaluación de la remoción de nitrógeno, fósforo y sulfuros en agua residual doméstica, utilizando Phragmites australis en Bioreactores, doi: http://dx.doi.org/10.4067/S0718-07642015000600011, Información Tecnológica, 26(6), 89-98 (2015) 
Denk, T., Mohn, J., Decock, C., Lewicka, D., Harris, E., Butterbach, K., Kiese, R. y Wolf, B., The nitrogen cycle: A review of isotope effects and isotope modeling approaches, doi: http://dx.doi.org/10.1016/j.soilbio.2016.11.015, Soil Biology \& Biochemistry, 105, 121-137 (2017)

Díaz-Cuenca, E., Alavarado-Granados, A.R. y Camacho-Calzada, K.E., El tratamiento de agua residual doméstica para el desarrollo local sostenible: el caso de la técnica del sistema unitario de tratamiento de aguas, nutrientes y energía (SUTRANE) en San Miguel Almaya, México, https://goo.gl/9RQ8QE; ISSN: 1405-8626, Quivera, 14(1), 78-97 (2012)

Elias, S., Mohamed, M., Ankur, A.N., Muda, K., Hassan, M., Othman, M.N. y Chelliapan, S., Water hyacinth Bioremediation for ceramic industry wastewater treatment application of Rhizofiltration system, https://goo.gl/dsQnKf; ISSN: 01266039, Sains Malaysiana, 43(9), 1397-1403 (2014)

Fonkou, T., Fonteh, M.F., Kanouo, M.D. y Amougou, A., Performances des filtres plantés de Echinochloa pyramidalis dans l'épuration des eaux usées de distillerie en Afrique subsaharienne, http://www.tropicultura.org/text/v28n2.pdf; ISSN: 07713312, Tropicultura, 28(2), 69-76 (2010)

González-Cabrera, O., Ruiz-Pérez, T., Claro-Pérez, M., Pérez-Pino, N.M., Pérez-Gálvez, G. y Collazo-Alfonso, L., Estudio de patentes sobre tecnologías para tratamiento de agua y el agua residual, doi: http://dx.doi.org/10.1590/010337862014000300010, TransInformação, 26(3), 339-347 (2014)

Gupta, P., Roy, S. y Mahindrakar, A.B., Treatment of water using water hyacinth, water lettuce and vetiver grass-A, doi: http://dx.doi.org/10.5923/j.re.20120205.04, Resources and Environment, 2(5), 202-215 (2012)

Hernández-Padilla, F., Margni, M., Noyola, A., Guereca-Hernandez, L. y Bulle, C., Assessing wastewater treatment in Latin America and the Caribbean: Enhancing life cycle assessment interpretation by regionalization and impact assessment sensibility, doi: http://dx.doi.org/10.1016/j.jclepro.2016.11.068, Journal of Cleaner Production, 142(4), 2140$2153(2017)$

Hidalgo, M. y Mejía, E., Diagnóstico De La Contaminación Por Aguas Residuales Domésticas, Cuenca Baja De La Quebrada La Macana, San Antonio De Prado. Municipio De Medellín. Tesis Especialistas, Facultad de Ingeniería, Universidad de Antioquia, Medellín-Colombia (2010)

Kipasika, H.J., Buza, J., Smith, W.A. y Njau, K.N., Removal capacity of faecal pathogens from wastewater by four wetland vegetation: Typha latifolia, Cyperus papyrus, Cyperus alternifolius and Phragmites australis, doi: http://dx.doi.org/10.5897/AJMR2016.7931, African Journal of Microbiology Research, 10(19), 654-661 (2016)

Lekeufack, M., Fonkou, T., Pamo, T. y Amougou, A., Removal of faecal bacteria and nutrients from domestic wastewater in a horizontal surface flow wetland vegetated with Echinochloa pyramidalis, doi: http://dx.doi.org/10.5897/AJEST12.126, African Journal of Environmental Science and Technology, 6(9), 337-345 (2012)

Mendoza, Y., Castro, F., Marín, J. y Behling, E., Phytoremediation as an alternative for domestic wastewater treatment from Riohacha City (Colombia), Revista Técnica de Ingeniería de la Univ. de Zulia, (39)2, 71-79 (2016)

Mudassar, F., Muhammad, I., Muhammad, F., Zeshan, A; Egrinya, E., Naureen, A., Ashiq, M. y Barkat, A., Effect of Cyclic Phytoremediation with Different Wetland Plants on Municipal Wastewater, doi: http://dx.doi.org/10.1080/15226514.2013.798623, International J. of Phytoremediation, 16(6), 572-581 (2014)

Mumtaz, S., Hashim, N., Arshad, A. y Abdul, R., Performance assessment of aquatic macrophytes for treatment of municipal wastewater, doi: http://dx.doi.org/10.1186/2052-336X-12-106, Journal of Environmental Health Science \& Engineering, 12(106), 1-12 (2014)

Niveth, C., Subraja, S., Sowmya, R. y Induja, N.M., Water Lettuce for Removal of Nitrogen and Phosphate from Sewage, doi: http://dx.doi.org/10.9790/1684-130201104107, Journal of Mechanical and Civil Engineering (IOSR-JMCE), 13(2), 104107 (2016).

Olguín, E., García-López, D., González-Portela, R. y Sánchez-Galván, G. Year-round phytofiltration lagoon assessment using Pistia stratiotes within a pilot-plant scale biorefinery, doi: http://dx.doi.org/10.1016/j.scitotenv.2017.03.067, Science of the Total Environment, 592, 326-333 (2017)

Priyanka, S., Omkar, S. y Supriya, S., Phytoremediation of industrial mines wastewater using water hyacinth, doi: http://dx.doi.org/10.1080/15226514.2016.1216078, Inter. Journal of phytoremediation, 19(1), 87-96 (2017)

Rezania, S., Ponraj, M., Din, M.F.M., Chelliapan, S. y Sairan, F.M., Effectiveness of Eichhornia crassipes in nutrient removal from domestic wastewater based onits optimal growth rate, doi: http://doi/abs/10.1080/19443994.2014.967305, Desalination and Water Treatment, 57, 360-365 (2014)

Rezania, S., Ponraj, M.; Talaiekhozani, A., Mohamad, E.S.; Din, M.F.M., Taib, S.M., Sabbagh, F. y Sairan, F.M., Perspectives of phytoremediation using water hyacinth for removal of heavy metals, organic and inorganic pollutants in wastewater, doi: http://dx.doi.org/10.1016/j.jenvman.2015.08.018, Journal of Environmental Management, 163, 125-133 (2015)

Rezania, S., Din, M.F.M., Taib, S., Dahalan, F., Songip, A., Singh, L. y Kamyab, H. The Efficient Role of Aquatic Plant (Water Hyacinth) in Treating Domestic Wastewater in Continuous System, doi: 10.1080/15226514.2015.1130018, International Journal of Phytoremediation, 18 (7), 679-685 (2016)

Rojano, R., Mendoza, Y., Arregoces, H. y Restrepo, G., Dispersión de PM10, NO2, CO, COV y HAP emitidos desde una Estación Modular de Compresión, Tratamiento y Medición de Gas Natural, doi: http://dx.doi.org/10.4067/S071807642016000400020, Inf. Tec. 27(5), 1-10 (2016) 
Romero, M., Colín, A., Sánchez, E. y Ortiz, M., Tratamiento de aguas residuales por un sistema piloto de humedales artificiales: evaluación de la remoción de la carga orgánica. UNAM, https://goo.gl/8HByms; ISSN: 0188-4999, Revista Internacional de Contaminación Ambiental, 25(3), 157-167 (2009)

Sánchez, R., Evaluación del humedal artificial de la hostería Cuicocha, utilizado para el tratamiento de aguas residuales domésticas. Tesis de pregado, Facultad de Ingeniería Civil y Ambiental, Universidad Politécnica Nacional, Quito, Ecuador (2011)

Sundaralingam, T. y Gnanavelrajah, N., Phytoremediation Potential of Selected Plants for Nitrate and Phosphorus from Ground Water, doi: http://dx.doi.org/10.1080/15226514.2013.773279, Int. J. Phytoremediation, 16(3), 275-284 (2014)

Tangahu, B.V., Abdullah, S.R.S., Basri, H., Idris, M., Anuar, N. y Mukhlisin, M., A review on heavy metals (As, Pb, and Hg) uptake by plants through phytoremediation, doi: http://dx.doi.org/10.1155/2011/939161, International Journal of Chemical Engineering, 1-31 (2011)

Valipour, A., Hamnabard, N., Woo, K.S. y Ahn Y.H., Performance of high-rate constructed phytoremediation process with attached growth for domestic wastewater treatment: Effect of high TDS and Cu, doi: http://dx.doi.org/10.1016/j.jenvman.2014.06.009, Journal of Environmental Management, 145, 1-8 (2014)

Valipour, A., Raman, V. y Ahn, Y., Effectiveness of domestic wastewater treatment using a bio-hedge water hyacinth wetland system, doi: http://dx.doi.org/10.3390/w7010329, Water, 7, 329-347 (2015)

Vangronsveld, J., Herzig, R., Weyens, N., Boulet, J., Adriaensen, K., Ruttens, A., Thewys, T., Vassilev, A., Meers, E., Nehnevajova, E., van der Lelie, D. y Mench, M., Phytoremediation of contaminated soils and groundwater: lessons fromthe field, doi: http://dx.doi.org10.1007/s11356-009-0213-6, Environmental Science and Pollution, 16(7), 765-794 (2009)

Vera, A., Andrade, C., Flores, E., Núñez, M., Cárdenas, C y Morales, E., Remoción de nutrientes y materia orgánica en un humedal construido en función del desarrollo de la macrófita Typha dominguensis Pers, Revista Técnica de la Facultad de Ingeniería Universidad del Zulia, 33(2), 153-163 (2010)

Vo, T-D., Bui, X-T., Nguyen, D-D., Nguyen, V-T., Ngo, H-H., Guo, W., Nguyen, P-D., Nguyen, C-N. y Lin, C., Wastewater treatment and biomass growth of eight plants for shallow bed wetland roofs, doi: https://doi.org/10.1016/j.biortech.2017.09.194, Bioresource Technology, 247, 992-998 (2017)

Vymazal, J. y Kröpfelová, L., Removal of organic in constructed wetlands with horizontal sub-surface flow: A review of the field experience, doi: https://doi.org/10.1016/j.scitotenv.2008.08.032, Science of the total environment, 407(13), 3911-3922 (2009)

Vymazal, J., Long-term performance of constructed wetlands with horizontal sub-surface flow: Ten case studies from the Czech Republic, doi: https://doi.org/10.1016/j.ecoleng.2009.11.028, Ecological Engineering, 37, 54-63 (2011)

Yousefi, Z. y Mohseni-Bandpei, A., Nitrogen and phosphorus removal from wastewater by subsurface wetlands planted with Iris pseudacorus, doi: https://doi.org/10.1016/j.ecoleng.2010.02.002, Ecological Engineering, 36, 777-782 (2010) 